\title{
Modified Seldinger technique for internal jugular open central venous line insertion in neonates: merging two different manoeuvers
}

\author{
Mohamed Mahmoud Shalaby* ${ }^{*}$, Rami Mohammed Salama and Mohammed Awad Mansour
}

\begin{abstract}
Background: Central venous line insertion in neonates is an important and lifesaving procedure. It can carry significant risks and complications, including death, at the time of insertion or later. We aimed to retrospectively assess the modified Seldinger technique for open placement of a central venous catheter in neonates, regarding its safety, feasibility, operative time, and preservation of the patency of the internal jugular vein. This study was conducted on 120 neonates from March 2018 to March 2020. We closely monitored the pulse for the detection of arrhythmia or bradycardia, which might be caused during the insertion of the guide wire or the tip of the catheter. Post-operative X-ray was done immediately after the end of the procedure for all cases, to determine the site of the central venous catheter and to detect the presence of pneumothorax.
\end{abstract}

Results: Arrhythmia was observed in 9 cases (7.5\%), and blood oozing in 5 cases (4.1\%). There were 3 cases of pneumothorax (2.5\%), 2 cases of neck hematoma in two cases (1.6\%), 6 cases of internal jugular vein thrombosis (5\%), and dislodging of the catheter in 3 cases (2.5\%). There were no cases of arterial puncture, failure of cannulation, or haemothorax in our study.

Conclusions: The modified Seldinger technique insertion for open central venous line in neonates is a safe, accessible, and feasible method, especially in centers that lack the experience of ultrasound-guided insertion in neonates.

Keywords: Seldinger, Venous, Lines, Neonates, Manoeuvers

\section{Background}

Central venous catheterization $(\mathrm{CVC})$ is an essential procedure in the neonatal intensive care unit, for the administration of life-saving treatments including total parenteral nutrition, nutritional support, and intravenous medication [1]. Veins such as the external jugular veins, internal jugular veins (IJV), subclavian veins, and femoral veins, are suitable sites for insertion of $\mathrm{CVC}$, as direct access for injection or infusion of different medications [2].

Despite the importance of CVC insertion, it carries significant risks and multiple complications (including

\footnotetext{
*Correspondence: mohamedmshalaby@gmail.com

Unit of Pediatric Surgery, Faculty of Medicine, Tanta University, Building

No. 33, Almadina Almonawra St., Shober Road, Tanta, Egypt
}

death) at the time of insertion or later on [3]. Many techniques have been developed for the insertion of CVC, but ultrasound-guided percutaneous CVC insertion allows quicker and safer vascular access in children. With regards to neonates, there is a limited experience for performing this technique in most institutions, and its performance is a challenge [4].

Right internal jugular vein catheterization in neonates has many difficulties and complications, even with ultra-sounded guided insertion of CVC, due to the small diameters of veins, collapsing of veins during closed punctures, and low birth weight of the neonates. Open surgical CVC insertion is a possible option in this case [5].

In this study, we aimed to assess retrospectively the modified Seldinger technique for open placement of a 
central venous catheter in neonates, as regards to its safety, feasibility, operative time, preservation of the patency of the internal jugular vein without its ligation, and the complications of this technique including pneumothorax, arrhythmia, fluid leakage, and thrombosis or dislodging of the central venous catheter. To our knowledge, this is the first study to merge these two techniques in neonates.

\section{Methods}

This retrospective study was conducted on 120 neonates in the neonatal intensive care unit (NICU) and pediatric surgery tertiary center from March 2018 to March 2020. We included all the cases that had central venous catheter insertion in the right internal jugular vein for medical or surgical causes, after failure of PICCs line insertion. We excluded femoral or subclavian CVCs insertion and patients with thrombosed internal jugular vein, also we excluded left-sided internal jugular line insertion to avoid anatomical factor. The parent or guardians of all participants provided written informed consent. Preoperative complete blood count $(\mathrm{CBC})$ and coagulation profile were done routinely for all cases. Doppler ultrasound on the neck veins was done pre-operatively in redo cases, and 2 weeks after catheter removal in all cases for assessment of the patency of veins. A chest X-ray was done immediately after CVC insertion, to detect the site of the catheter tip and any complications.

\section{The technique}

For all our cases (120 neonates), we inserted the catheters in the right internal jugular vein (IJV). All patients had general anesthesia or sedation. Insertion was performed in either the operating room, or in the NICU. Bedside cases received both sedation and local anesthesia. The patients were positioned in $30^{\circ}$ (Trendelenburg position), with a roll under their shoulders for neck extension. The patient's neck was rotated to the left side to expose the surgical incision site. Under full sterile technique, a transverse incision was made on the right side of the neck, in the middle of the triangle between the two heads of the sternocleidomastoid muscle, and the superior border of medial third of the clavicle. The sternal part of the sternocleidomastoid muscle is retracted medially, and dissection is made between the sternal and clavicular heads. The right internal jugular vein was dissected and identified from the internal carotid artery. After proximal and distal control of the vein, a 24-G. cannula was carefully inserted directly to the IJV. The guidewire was inserted through the cannula. We assured the site of the guidewire with the aid of $\mathrm{C}$-arm in the operating room, or $\mathrm{x}$-ray in the NICU, then the cannula was removed. Through a subcutaneous tunnel, a size 4 French double lumen short term catheter was passed and brought out through it. Radiographic assessment was performed to ensure catheter tip location and optimal depth of catheter insertion was guided by the common equation [length of insertion $(\mathrm{cm})=1.7+(0.07 \times$ height $)]$, after which the guidewire was removed. A 4/0 silk suture was placed on the skin to fix the line at the exit site, and the catheter was flushed with heparin (10 units per $1 \mathrm{ml}$ normal saline). The wound was closed after the correct catheter position and good hemostasis was obtained; the area was covered with sterile dressing (i.e., modified Seldinger technique) (Figs. 1 and 2).

During the procedure, the pulse was monitored for the detection of arrhythmia or bradycardia, which might be caused during the insertion of the guidewire or the tip of the catheter. Post-operative X-ray were done immediately at the end of the procedure for all cases, to determine the site of CVC and to detect the presence of pneumothorax.

Two weeks after of catheter removal (including discharged cases), Doppler ultrasound of the neck was requested to assess the patency of the neck veins.

\section{Statistics}

Descriptive statistics were reported for sample data. Categorical variables were summarized using frequency and percentages. Mean and standard deviation was used to describe continuous variables. All analysis was performed in SPSS (version 26).

\section{Results}

One hundred twenty neonates were included in this retrospective study. The study was conducted in the NICU and pediatric surgery unit in our university hospitals from March 2018 to March 2020. Right IJV catheter was performed in all neonates, inserted by the open modified Seldinger technique. The indications for line insertion were mainly for total parenteral nutrition, intravenous fluids, and medications in cases with difficult cannulation.

Out of 120 cases, 75 cases were males while 45 cases were females. Redo cases were recorded in 24 patients after planned removal and line insertion was re-indicated again with time interval (mean $\pm \mathrm{SD})(7 \pm 2.82)$ days between the first line and the reinserted one. Neonate weights were 3 cases less than $1 \mathrm{~kg}, 2$ cases were from 1 to $1.5 \mathrm{~kg}, 71$ cases were from $1.5 \mathrm{~kg}$ to $2 \mathrm{~kg}, 34$ cases were from $2 \mathrm{~kg}$ to $2.5 \mathrm{~kg}, 5$ cases were from $2.5 \mathrm{~kg}$ to $3 \mathrm{~kg}$ and 5 cases were more than $3 \mathrm{~kg}$. The minimum weight was 750 $\mathrm{g}$, while the maximum weight of our studied cases was $3700 \mathrm{~g}$ (Table 1).

The age of the patients at the time of insertion of CVC were within the first week of life in 54 cases, 38 cases were in the second week of life, 18 cases were during the 


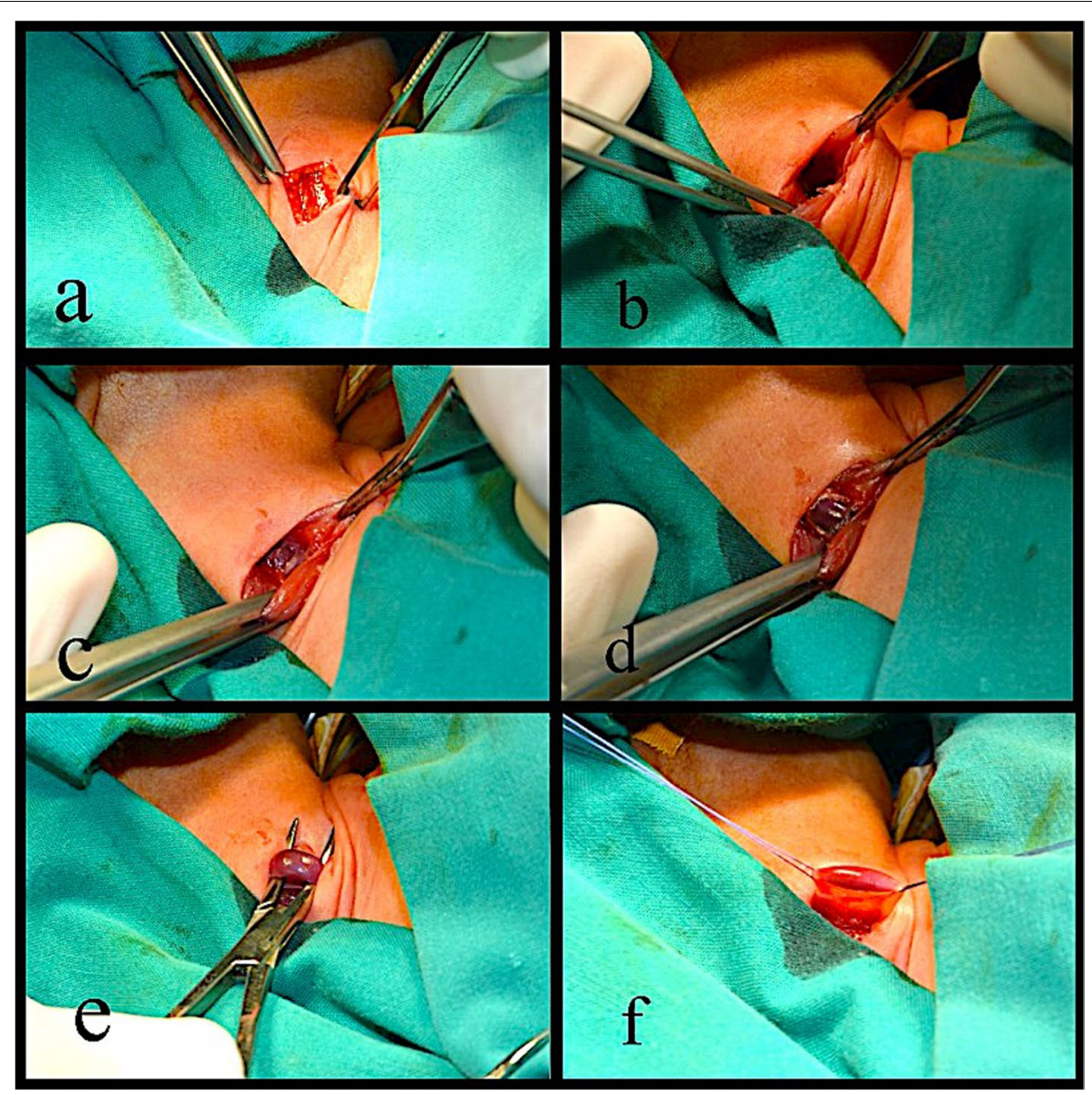

Fig. 1 Internal Jugular vein dissection. a Skin incision. b Muscle retracted. c Fascia dissection. $\mathbf{d}$ Vein exposure. e Vein dissection. f Vein control

third week of life, and 10 cases were in the fourth week of life (Table 2).

During the time of insertion of the CVC, 20 cases were on the nasal cannula, 88 cases were on low parameters ventilation, while 12 cases were on high parameters ventilation. The operative time of insertion of CVC ranged from 15 min to 35 min (mean \pm SD) $(23.71 \pm 3.82)$.

Intraoperative close monitoring of the patients was performed in our study; 9 cases $(7.5 \%)$ developed temporary arrhythmia at the time of insertion of the guidewire (corrected immediately after gradual withdrawal of the guidewire), and 5 cases (4.1\%) developed minute blood oozing after insertion of the CVC (this was stopped by compression). There were no events of arterial puncture or failure of cannulation in our study.

Immediately after insertion of the $\mathrm{CVC}$, a chest X-ray chest for the neonates was performed to detect the position of the tip of catheters, which was in the right position for all our patients. Pneumothorax was detected in 3 cases $(2.5 \%)$ which was mild; in two of the cases, it was resolved spontaneously, while one patient required a chest tube. Two (1.6\%) cases developed neck hematoma within the first two days after $\mathrm{CVC}$ insertion, which resolved spontaneously. Breaking or leaking of the catheter was detected in 2 cases (1.6\%), five cases developed infection (4.1\%). Local infection was reported in five cases. It happened in the second week of insertion in 2 patients and in the third week of insertion in 3 patients. There were no cases of leakage from the tunnel. The removal of the catheter (depending on neonates' requirements) ranged from 6 days to 25 days from the date of insertion (mean $\pm \mathrm{SD})(16.47 \pm 3.38)$.

Dislodging of the CVC was recorded in 3 cases after 8,10 , and 13 days after insertion respectively. No cases required reinsertion as they did not require central catheterization. We routinely requested Doppler ultrasound of their neck veins to assess their patency after 2 weeks from the time of CVC removal, and even for discharged cases. Eight cases out of 120 studied cases died by medical or surgical causes unrelated to $\mathrm{CVC}$ insertion or $\mathrm{CVC}$ complications. Doppler assessments revealed patent neck veins on the side of the previously inserted CVC in 106 


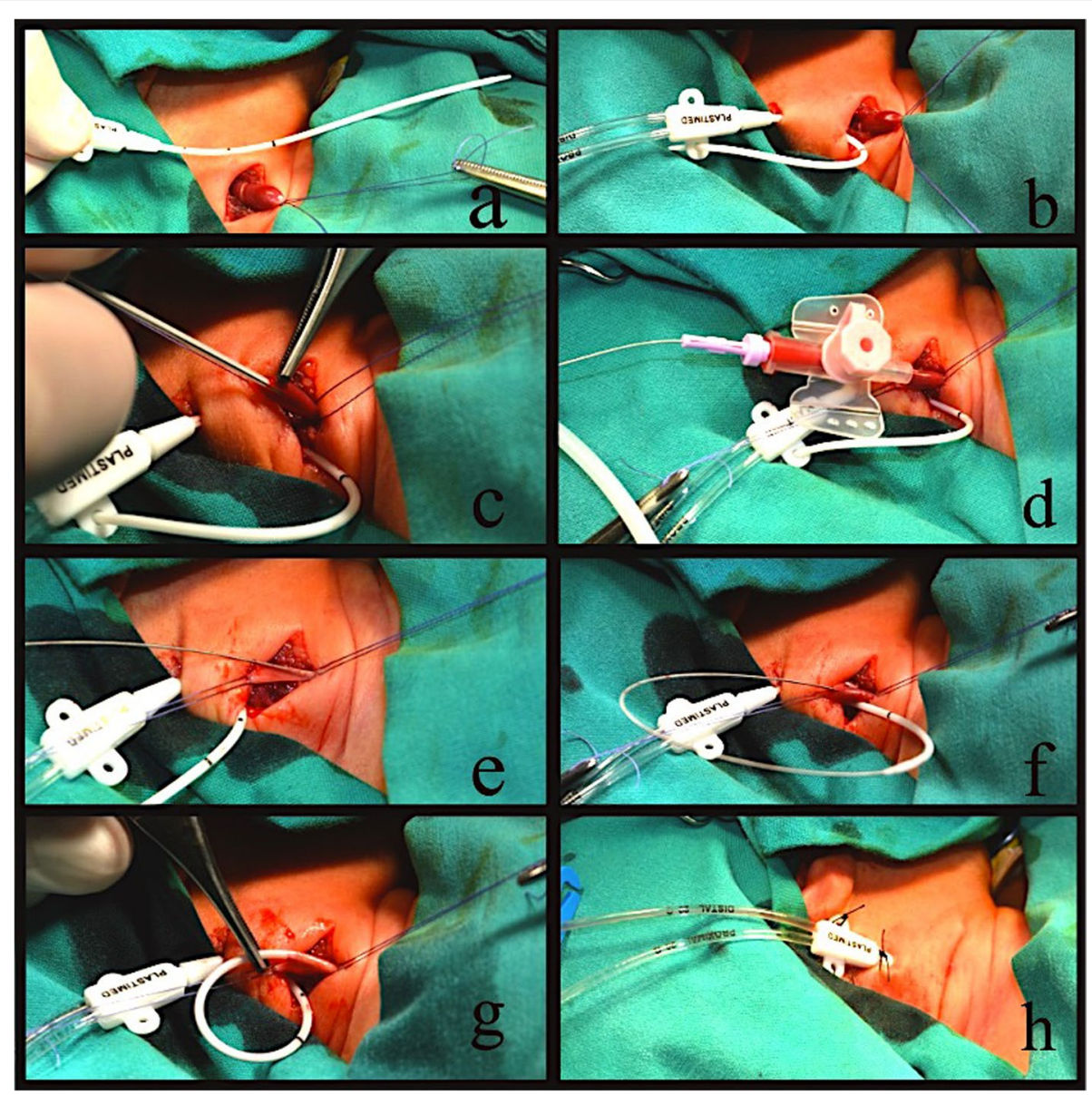

Fig. 2 Line insertion. a Checking the line. b Subcutaneous tunnel. c Cannulation. $\mathbf{d}$ Guide wire insertion. e Cannula out. $\mathbf{f}$ Insertion of the line over the guidewire. $\mathbf{g}$ Advancing the line. $\mathbf{h}$ Line fixation

Table 1 The weight of the neonates at the time of CVC insertion

\begin{tabular}{ll}
\hline Weight of the neonate $/ \mathbf{k g}$ & No. of cases $(\boldsymbol{n}=\mathbf{1 2 0})$ \\
\hline Less than $1 \mathrm{~kg}$ & $3(2.5 \%)$ \\
$1-1.5 \mathrm{~kg}$ & $2(1.6 \%)$ \\
$1.5-2 \mathrm{~kg}$ & $71(59.1 \%)$ \\
$2-2.5 \mathrm{~kg}$ & $34(28.3 \%)$ \\
$2.5-3 \mathrm{~kg}$ & $5(4.1 \%)$ \\
More than $3 \mathrm{~kg}$ & $5(4.1 \%)$ \\
\hline
\end{tabular}

Table 2 Age of the neonates at the time of CVC insertion

\begin{tabular}{ll}
\hline Age of the neonates & No. of cases $(\boldsymbol{n}=\mathbf{1 2 0})$ \\
\hline $0-7$ days & $54(45 \%)$ \\
$8-14$ days & $38(31.6 \%)$ \\
$15-21$ days & $18(15 \%)$ \\
$22-28$ days & $10(8.3 \%)$ \\
\hline
\end{tabular}

cases (95\%) out of 112 cases ( excluding 8 mortality cases), while 6 cases $(5 \%)$ revealed thrombosis of IVJ in the side of the previously inserted CVC. All complications were summarized in Table 3.

\section{Discussion}

Central venous lines insertion is one of the commonest procedures in major tertiary-level pediatric hospitals. Current practice in the utilization of CVC for patients receiving care in the NICU is not well elucidated. CVC often involves a dedicated surgical team, and serious complications, while rare, do exist [6-9].

In 2009, Kaji et al. began to perform US-guided central venous catheterization into the IJV. Providing the US probe is placed at the summit of the triangle formed by the two subdivisions of the sternocleidomastoid muscle and the clavicular bone, the IJV and common carotid artery can be identified. Real-time US imaging might cause operators to assume that the IJV puncture is easy. The 
Table 3 Summary of complications

\begin{tabular}{lll}
\hline Complications & & No. of cases $(\boldsymbol{n}=\mathbf{1 2 0})$ \\
\hline Intra-operative & Arrhythmia & $9(7.5 \%)$ \\
& Blood oozing & $5(4.1 \%)$ \\
& Arterial puncture & $0(0 \%)$ \\
& Failure to cannulation & $0(0 \%)$ \\
Post-operative & Pneumothorax & $3(2.5 \%)$ \\
& Hemothorax & $0(0 \%)$ \\
& Neck hematoma & $2(1.6 \%)$ \\
& I.J.V thrombosis & $6(5 \%)$ \\
& Dislodging of catheter & $3(2.5 \%)$ \\
& Breaking or leaking of the & $2(1.6 \%)$ \\
& catheter & \\
& Leak from tunnel & $0(0 \%)$ \\
Total & Infection & $5(4.1 \%)$ \\
& & $35(29.1 \%)$ \\
\hline
\end{tabular}

continuous visualization of the needle tip during needle advancement is quite difficult. Kaji et al. conducted USguided catheterization, with the probe placed in the short axis. Previous studies have compared the short- and longaxis approaches to vascular access, and have reported that needle tip visibility at the time of vessel puncture was higher with the long-axis approach (62\%) than with the short-axis approach (23\%) [10]. Thus, the short-axis approach is difficult, and the long-axis approach might be recommended. However, because the cervical space in children is not wide enough for the placement of the US probe, the long-axis approach is not acceptable for small children. Additionally, Blaivas reported that US-guided vascular access using the short-axis approach regularly penetrated the posterior wall of the IJV [11-13].

In our institution, as a tertiary center for neonatology and pediatric surgery, we adopted modified Seldinger technique for open central venous lines insertion in neonates (hybrid technique), as our anesthesia team had no experience with neonates in the ultrasound-guided percutaneous insertion of the central venous catheter.

Our intraoperative complications were arrhythmia in 9 cases $(7.5 \%)$, and blood oozing in 5 cases $4.1 \%$. We had no events of arterial puncture, or failure of cannulation. We assume that this was due to direct vision, and good control of the internal jugular vein. With regard to our postoperative complications, there were 3 cases of Pneumothorax (2.5\%), neck hematoma in 2 cases (1.6\%), IJV thrombosis in 6 cases $(5 \%)$ and dislodging of the catheter in 3 cases $(2.5 \%)$. There were no cases of haemothorax, as we did not insert in the subclavian veins, and we had good control of the internal jugular vein during insertion. Breaking or leaking of the catheter was detected in 2 cases (1.6\%), no cases had leak from the tunnel, and 5 cases developed infection (4.1\%) that was treated by antibiotics according to culture sensitivity.

The new technique saves time of venous dissection, venotomy, and control of bleeding and closure of venotomy. We faced less risk of bleeding as we avoid the vascular dissection and venotomy (which is the main steps of the routine open technique). For these reasons, it is easily to be learned and performed with short time learning curve. Postoperatively, this technique minimizes vein thrombosis and give chance for reinsertion in the same site over a guide wire like the advantage of closed method, this advantage is lacked in the routine open technique.

According to international literature, the overall complication rate in U/S-guided PCVC insertion in pediatric age ranges between 2.4 and $4.6 \%$. Avanzini et al. observed the complication rate in U/S-guided insertion per 1,000 catheter days. Their complications included 15 (7.7\%) mechanical incidents and 7 (3.6\%) cases of infection; however, the remaining $5(2.6 \%)$ were classified as intraoperative complications. However, this study only included pediatric cases, and did not specifically study the procedure in neonates. They explained their high rate of complications due to the learning curve of U/S-guided procedur e[14].

Basford et al, in their comparison of complication rates between surgical and radiologic placement, found higher rates of infectious and mechanical complications among surgically placed catheters, than those placed radiologically ( $47.1 \%$ vs $16.7 \%$ and $50.0 \%$ vs $16.7 \%$, respectively). Mean complications per 1000 days reflected this trend, but just failed to reach significance [15].

Arul G.S. et al. reported that many UK pediatric surgeons use open surgical cutdown (OSC) as the technique of choice; even those with an interest in using percutaneous landmark technique (LT) often prefer (OSC) in smaller babies requiring central venous cannulation [16].

This current study has limitations, which we recommend future studies to try to overcome. These include retrospective study design, single-center experience, and a small sample size. We recommend the adoption of our technique on a multicenter prospective scale, with a control group of closed percutaneous technique or ultrasound-guided group.

Our adopted technique (modified Seldinger maneuver) is safe, and feasible especially in neonates, and provides easy and accurate detection of the internal jugular vein.

\section{Conclusion}

The modified Seldinger technique insertion of the open central venous line in neonates is a safe, accessible, and feasible method especially in centers that lack the experience of ultrasound-guided percutaneous $\mathrm{CVC}$ insertion in neonates. 


\section{Abbreviations}

CVC: Central venous catheter; IJV: Internal jugular veins; NICU: Neonatal intensive care unit; CBC: Complete blood count; SD: Standard deviation; u/s: Ultra-sound; OSC: Open surgical cutdown; LT: Landmark technique; PCVC: Pediatric central venous catheter.

\section{Acknowledgements}

Not applicable

\section{Authors' contributions}

MS designed the research, collected, analyzed, interpreted the patient data, and wrote the manuscript. RS assisted in data collection and in writing. MA collected the data and was a major contributor in writing the manuscript. All authors read and approved the final manuscript.

\section{Funding}

This study was not funded.

\section{Availability of data and materials}

The datasets used and/or analyzed during the current study are available from the corresponding author on reasonable request.

\section{Declarations}

\section{Ethics approval and consent to participate}

This study was approved by the Research Ethics Committee of Tanta University, Faculty of Medicine with approval number [c32971/12/2019]. The parent or guardians of all participants provided written informed consent.

\section{Consent for publication}

Not applicable

\section{Competing interests}

The authors declare that they have no competing interests.

Received: 2 October 2020 Accepted: 10 October 2021

Published online: 13 December 2021

\section{References}

1. Vegunta RK, Loethen P, Wallace LJ, Albert VL, Pearl RH (2005) Differences in the outcome of surgically placed long-term central venous catheters in neonates: neck vs groin placement. J. Pediatr. Surg. 40:47-51. https:// doi.org/10.1016/j.jpedsurg.2004.09.015

2. Merrer J, De Jonghe B, Golliot F, Lefrant JY, Raffy B, Barre E, Rigaud JP, Casciani D, Misset B, Bosquet C, Outin H, Brun-Buisson C, Nitenberg G (2001) Complications of femoral and subclavian venous catheterization in critically ill patients: a randomized controlled trial. JAMA. 286:700-707. https://doi.org/10.1001/jama.286.6.700

3. Kusminsky RE (2007) Complications of central venous catheterization. J. Am. Coll. Surg. 204:681-696. https://doi.org/10.1016/j.jamcollsurg.2007. 01.039

4. Detaille T, Pirotte T, Veyckemans F (2010) Vascular access in the neonate. Best Pract. Res. Clin. Anaesthesiol. 24:403-418. https://doi.org/10.1016/j. bpa.2010.02.017

5. Morita M, Sasano H, Azami T, Sasano N, Fujita Y, Ito S, Sugiura T, Sobue K (2009) A novel skin-traction method is effective for real-time ultrasound-guided internal jugular vein catheterization in infants and neonates weighing less than 5 kilograms. Anesth. Analg. 109:754-759. https://doi.org/10.1213/ane.0b013e3181b01ae3

6. Fratino G, Avanzini S, Molinari AC, Buffa P, Castagnola E, Haupt R (2009) Incidence of indwelling central venous catheter-related complications using the Sri Paran technique for device fixation in children with cancer. Pediatr. Surg. Int. 25:591-594. https://doi.org/10.1007/s00383-009-2400-4

7. Journeycake JM, Buchanan GR (2003) Thrombotic complications of central venous catheters in children. Curr. Opin. Hematol. 10:369-374 https://doi.org/10.1097/00062752-200309000-00008

8. Castagnola E, Molinari AC, Giacchino M, Chiapello N, Moroni C, Caviglia I, Fratino G, Haupt R (2007) Incidence of catheter-related infections within 30 days from insertion of Hickman-Broviac catheters. Pediatr. Blood Cancer. 48:35-38. https://doi.org/10.1002/pbc.20724

9. Sheridan RL, Weber JM (2006) Mechanical and infectious complications of central venous cannulation in children: lessons learned from a 10-year experience placing more than 1000 catheters. J. Burn Care Res. Off. Publ. Am. Burn Assoc. 27:713-718. https://doi.org/10.1097/01.BCR.0000238087. 12064.E0

10. Stone MB, Moon C, Sutijono D, Blaivas M (2010) Needle tip visualization during ultrasound-guided vascular access: short-axis vs long-axis approach. Am. J. Emerg. Med. 28:343-347. https://doi.org/10.1016/j.ajem. 2008.11.022

11. Blaivas M, Adhikari S (2009) An unseen danger: frequency of posterior vessel wall penetration by needles during attempts to place internal jugular vein central catheters using ultrasound guidance. Crit. Care Med. 37:2345-2349; quiz 2359. https://doi.org/10.1097/CCM.0b013e3181 $\mathrm{a} 067 \mathrm{~d} 4$

12. French JLH, Raine-Fenning NJ, Hardman JG, Bedforth NM (2008) Pitfalls of ultrasound guided vascular access: the use of three/four-dimensional ultrasound. Anaesthesia. 63:806-813. https://doi.org/10.1111/j.1365-2044 2008.05513.x

13. Kaji T, Kawano T, Yamada W, Yamada K, Onishi S, Nakame K, Mukai M, leiri S, Takamatsu H (2016) The changing profile of safe techniques for the insertion of a central venous catheter in pediatric patients - improvement in the outcome with the experiences of 500 insertions in a single institution. J. Pediatr. Surg. 51:2044-2047. https://doi.org/10.1016/j.jpedsurg. 2016.09.037

14. Avanzini S, Guida E, Conte M, Faranda F, Buffa P, Granata C, Castagnola E, Fratino G, Mameli L, Michelazzi A, Pini-Prato A, Mattioli G, Molinari AC, Lanino E, Jasonni V (2010) Shifting from open surgical cut down to ultrasound-guided percutaneous central venous catheterization in children: learning curve and related complications. Pediatr. Surg. Int. 26:819-824. https://doi.org/10.1007/s00383-010-2636-z

15. Basford TJ, Poenaru D, Silva M (2003) Comparison of delayed complications of central venous catheters placed surgically or radiologically in pediatric oncology patients. J. Pediatr. Surg. 38:788-792. https://doi.org/ 10.1016/jpsu.2003.50168

16. Arul GS, Lewis N, Bromley P, Bennett J (2009) Ultrasound-guided percutaneous insertion of Hickman lines in children. Prospective study of 500 consecutive procedures. J. Pediatr. Surg 44:1371-1376. https://doi.org/10. 1016/j.jpedsurg.2008.12.004

\section{Publisher's Note}

Springer Nature remains neutral with regard to jurisdictional claims in published maps and institutional affiliations. 